\title{
A General Framework for Splitting Concepts for Cocycles over Generalized Nonautonomous Dynamical Systems
}

\author{
Codruţa Stoica \\ Department of Mathematics and Computer Science, Aurel Vlaicu University of Arad, Arad, Romania \\ Correspondence should be addressed to Codruţa Stoica; codruta.stoica@uav.ro
}

Received 15 October 2016; Accepted 4 January 2017; Published 26 January 2017

Academic Editor: Hiroyuki Mino

Copyright (C) 2017 Codruţa Stoica. This is an open access article distributed under the Creative Commons Attribution License, which permits unrestricted use, distribution, and reproduction in any medium, provided the original work is properly cited.

\begin{abstract}
While the classic theory of exponential dichotomy deals with differential and difference equations with uniquely determined forward and backward solutions, nowadays, applications in engineering require having corresponding theory for equations whose backward solutions are not guaranteed to exist or to be unique. To this goal, we will consider general dichotomic behaviors that consist in assuming the existence of splitting into invariant subspaces, where the norms of the evolution trajectories are bounded by some functions that depend on the initial and final times.
\end{abstract}

\section{Introduction}

Several issues emphasized by Daleckii and Krein at the 5th International Congress of Nonlinear Oscillations, held in Kiev in 1969, have focused on the necessity of the study of asymptotic behaviors of differential equations with bounded coefficients on Banach spaces. This important step in the development of the stability theory consists in a natural launch in the study of evolution equations given by unbounded operators. Also, the approach by means of associated operator families has allowed obtaining answers to some open problems by involving techniques of functional analysis and operator theory. The importance of the evolution semigroups in the study of dynamical systems was pointed out by Chicone and Latushkin in [1] and the dynamics of evolutionary equations by Sell and You in [2].

One of the central interests in the study of asymptotic behaviors of dynamical systems is to find conditions for their solutions to be stable, unstable, or dichotomic. The exponential dichotomy is one of the basic concepts arising in the theory of dynamical systems which describe phenomena from real world. It was introduced by Perron in 1930, in [3], as he was concerned with the problem of conditional stability of an equation $\dot{x}=A(t) x$ and the connection with the existence of bounded solutions of the equation $\dot{x}=A(t) x+f(t, x)$. The papers of Perron served as a starting point for many works on the stability theory.

This topic plays a central role in the study of stable and unstable manifolds and in many aspects of the theory of stability. Even in the context of bifurcation theory, the exponential dichotomy has a role. The exponential dichotomy has gained prominence since the appearance of two fundamental monographs due to Daleckii and Krein (see [4]) and Massera and Schäffer (see [5]). Other remarkable results were obtained by Latushkin et al. in [6] and Sacker and Sell in [7].

While the basic theory of exponential dichotomies deals with differential and difference equations with uniquely determined forward and backward solutions, nowadays, applications require having a corresponding theory for equations whose backward solutions are not guaranteed to exist or to be unique.

The exponential splitting of order $n$ was studied in [4], where Daleckii and Krein established that the operator $L=$ $d / d t+A$, with bounded operator-function $A$, which admitted exponential splitting, was stable with respect to perturbations small in norm.

The study in discrete time, done by Aulbach, Kalkbrenner, and Siegmund in $[8,9]$ or $[10]$, answered the question of how to define a suitable notion of exponential dichotomy for difference equations whose right-hand sides are not supposed 
to be invertible. To this end, they have defined the exponential forward splitting. Only this concept was not satisfactory as it did not provide a standard perturbation result, compulsory in any theory of dichotomies. Hence, they added a regularity condition, which solved the problem.

In a discrete setting, the relative asymptotic equivalence between difference equations was studied by Leiva et al. in [11], where the definition of the discrete dichotomy is one of the important tools in obtaining the results.

The concept of evolution operators arises naturally in engineering from the theory of well-posed nonautonomous Cauchy problems, while the notion of linear skew-product semiflows is involved when considering the linearization along an invariant manifold of a dynamical system generated by a nonlinear differential equation, as described by Chow and Leiva in [12]. This study originated in the ergodic theory of discrete dynamical systems, but, later, continuous skewproduct flows have provided a unified topological way to study the dynamics of nonautonomous differential equations.

The skew-evolution semiflows, defined in [13], are generalizations for both evolution operators and skew-product semiflows.

Various asymptotic behaviors for skew-evolution semiflows, as stability, instability, dichotomy, and trichotomy, are studied in [14-18] or [19]. The connections between the dichotomy and splitting property were emphasized in [20]. The notion of skew-evolution semiflow was already adopted and its applicability emphasized by Bento and Silva (see [21]), Hai (see [22, 23]), and Yue et al. (see [24]).

In this paper, we study the problem of how to generalize the notion of exponential dichotomy to differential equations whose solutions are only assumed to exist in forward time. A straightforward generalization does not provide satisfactory results but, with additional assumptions, the goal can be reached. In [25], Chow and Leiva introduce a concept of exponential dichotomy for linear skew-product semiflows in infinite dimensional Banach spaces, as an extension for the classical notion of exponential dichotomy for timedependent linear differential equations. In [26], Leiva and Rodrigues study the relative asymptotic equivalence between two evolutions equations in a Banach space by means of a generalized notion of dichotomy.

This paper emphasizes a general framework for the exponential splitting that includes, as a particular case, the exponential dichotomy. It is induced on a Banach space by a skew-evolution cocycle (see [13]) defined over a semiflow associated with a generalized dynamical system. Both a nonautonomous equation and a variational equation can be addressed in terms of skew-evolution semiflows. We prove that the concept of exponential splitting is stronger than the notion of exponential dichotomy, but they can be equivalent under suitable conditions.

We will consider general dichotomic behaviors that consist in assuming the existence of splitting into invariant subspaces, where the norms of the evolution trajectories are bounded by some functions that depend on the initial and final times. The additional conditions concern the families of projectors and the exponential growth of the skew-evolution cocycle. Characterizations and connections, underlined by examples and counterexamples, are also provided.

The stable and the unstable subspaces of the phase state are described in terms of the boundedness of the corresponding projector along the evolution cocycle, forward and backward, and in terms of the exponential decay of the skew-evolution cocycle.

\section{Skew-Evolution Cocycles}

Let us fix some standard notations: $(X, d)$ is a metric space, $V$ is a Banach space, and $\mathscr{B}(V)$ is the space of all $V$-valued bounded operators defined on $V$. The norm of vectors on $V$ is denoted by $\|\cdot\|$. Let us consider $Y=X \times V$ and $\Delta=\left\{\left(t, t_{0}\right) \in\right.$ $\left.\mathbb{R}_{+}^{2}: t \geq t_{0}\right\} . I$ is the identity operator.

Definition 1. A map $\tilde{u}: \Delta \times X \rightarrow \mathscr{P}(X)$ with the properties,

$$
\begin{aligned}
& \left(s_{1}\right) \tilde{u}(t, t, \cdot)=i d_{X}, \forall t \in \mathbb{R}, \\
& \left(s_{2}\right) \tilde{u}(t, \tau, x) \subseteq \widetilde{u}(t, s, \widetilde{u}(s, \tau, x)), \forall(t, s),(s, \tau) \in \Delta, \forall x \in \\
& \quad X,
\end{aligned}
$$

is a generalized nonautonomous dynamical system on $X$.

Multivalued nonautonomous dynamical systems were studied, for example, by Caraballo et al. in [27] or by Valero in [28]. Some asymptotic properties for cocycles over dynamical systems are given in $[29,30]$.

Remark 2. In what follows, we will consider the case of a mapping $u: \Delta \times X \rightarrow X$ with the properties,

$$
\begin{aligned}
& \left(s_{1}^{\prime}\right) u(t, t, x)=x, \forall(t, x) \in \mathbb{R}_{+} \times X, \\
& \left(s_{2}^{\prime}\right) u\left(t, t_{0}, x\right)=u\left(t, s, u\left(s, t_{0}, x\right)\right), \forall(t, s),\left(s, t_{0}\right) \in \Delta, \forall x \in \\
& \quad X,
\end{aligned}
$$

called the evolution semiflow associated with the generalized nonautonomous dynamical system on $X$.

$$
\begin{aligned}
& \text { Definition 3. A mapping } U: \Delta \times X \rightarrow \mathscr{B}(V) \text { satisfying } \\
& \begin{array}{l}
\left(c_{1}\right) U(t, t, x)=I_{V}, \forall(t, x) \in \mathbb{R}_{+} \times X, \\
\left(c_{2}\right) U\left(t, s, u\left(s, t_{0}, x\right)\right) U\left(s, t_{0}, x\right)=U\left(t, t_{0}, x\right), \forall(t, s),(s, \\
\left.t_{0}\right) \in \Delta, \forall x \in X,
\end{array}
\end{aligned}
$$

is called skew-evolution cocycle over $u$.

Remark 4. If $U$ is a skew-evolution cocycle over $u$ and $\lambda \epsilon$ $\mathbb{R}$, then $U_{\lambda}: \Delta \times X \rightarrow \mathscr{B}(V)$, given by $U_{\lambda}(t, s, x)=$ $e^{-\lambda(t-s)} U(t, s, x)$, is the $\lambda$-shifted skew-evolution cocycle over $u$. The reason of the terminology shifted can be appreciated by assuming that if $U$ is a solution operator associated with the nonautonomous system $\dot{w}=A(t) w$, then $U_{\lambda}$ is also a solution operator associated with the shifted system $\dot{w}=$ $\left[A(t)-\lambda I_{V}\right] w$.

Example 5. $\mathscr{C}\left(\mathbb{R}_{+}, \mathbb{R}_{+}\right)$is the set of all continuous functions $x: \mathbb{R}_{+} \rightarrow \mathbb{R}_{+}$, endowed with the topology of uniform convergence on compact subsets of $\mathbb{R}_{+} . X \subset \mathscr{C}\left(\mathbb{R}_{+}, \mathbb{R}_{+}\right)$. The mapping $u: \Delta \times X \rightarrow X$, defined by $u(t, s, x)=x_{t-s}$, where 
$x_{t-s}: \mathbb{R}_{+} \rightarrow \mathbb{R}_{+}$is given by $x_{t-s}(\tau)=x(t-s+\tau)$, is an evolution semiflow on $X$.

Example 6. Let us consider $X=\mathbb{R}$ and $u$ as defined in Example 5. We consider $\Phi: \Delta \rightarrow \mathscr{B}(V)$ to be the evolution operator associated with the nonautonomous differential equation $\dot{x}=A(t) x, t \in \mathbb{R}$; that is, the solution $x(\cdot)$ satisfies the relation $x(\tau)=\Phi(\tau, s) x(s), \forall \tau \geq s$. The mapping $U_{\Phi}$ : $\Delta \times X \rightarrow \mathscr{B}(V)$, given by $U_{\Phi}(t, s, x)=\Phi(t-s+x, x)$, is a skew-evolution cocycle on $V$ over $u$.

Example 7. Let $f: \mathbb{R}_{+} \rightarrow \mathbb{R}_{+}^{*}$ be a decreasing function with the property that there exists $\lim _{t \rightarrow \infty} f(t)=a>0$. We denote by $\mathscr{C}=\mathscr{C}\left(\mathbb{R}_{+}, \mathbb{R}_{+}\right)$the set of all continuous functions $x: \mathbb{R}_{+} \rightarrow \mathbb{R}_{+}$, endowed with the topology of uniform convergence on compact subsets of $\mathbb{R}_{+}$, metrizable by means of the distance:

$$
\begin{aligned}
d(x, y)=\sum_{n=1}^{\infty} \frac{1}{2^{n}} & \frac{d_{n}(x, y)}{1+d_{n}(x, y)}, \\
& \text { where } d_{n}(x, y)=\sup _{t \in[0, n]}|x(t)-y(t)| .
\end{aligned}
$$

If $x \in \mathscr{C}$, then, for all $t \in \mathbb{R}_{+}$, we denote $x_{t}(s)=x(t+s)$, $x_{t} \in \mathscr{C}$. Let $X$ be the closure in $\mathscr{C}$ of the set $\left\{f_{t}, t \in \mathbb{R}_{+}\right\}$. It can easily be shown that $(X, d)$ is a metric space and the mapping $u: \Delta \times X \rightarrow X, u(t, s, x)=x_{t-s}$ is an evolution semiflow on $X$.

We consider $V=\mathbb{R}^{2}$, with the norm $\|v\|=\left|v_{1}\right|+\left|v_{2}\right|$, $v=\left(v_{1}, v_{2}\right) \in V$. If $g: \mathbb{R}_{+} \rightarrow \mathbb{R}_{+}^{*}$, then the mapping $U_{g}:$ $\Delta \times X \rightarrow \mathscr{B}(V)$, defined by

$$
\begin{aligned}
& U_{g}(t, s, x) v \\
& =\left(\frac{g(s)}{g(t)} e^{-\int_{s}^{t} x(\tau-s) d \tau} v_{1}, \frac{g(t)}{g(s)} e^{\int_{s}^{t} x(\tau-s) d \tau} v_{2}\right),
\end{aligned}
$$

is a skew-evolution cocycle over $u$.

Example 8. Let $X=\mathbb{R}_{+}$. The mapping $u: \Delta \times \mathbb{R}_{+} \rightarrow \mathbb{R}_{+}$given by $u(t, s, x)=t-s+x$ is an evolution semiflow on $\mathbb{R}_{+}$. For every evolution operator $E: \Delta \rightarrow \mathscr{B}(V)$ (i.e., satisfying the properties $E(t, t)=I, \forall t \in \mathbb{R}_{+}$, and $E(t, s) E\left(s, t_{0}\right)=E\left(t, t_{0}\right)$, $\left.\forall(t, s),\left(s, t_{0}\right) \in \Delta\right)$ the mapping $U_{E}: \Delta \times \mathbb{R}_{+} \rightarrow \mathscr{B}(V)$ defined by $U_{E}(t, s, x)=E(t-s+x, x)$ is a skew-evolution cocycle on $V$ over the evolution semiflow $u$. Hence, an evolution operator on $V$ is generating a skew-evolution cocycle on $Y$.

Other examples of skew-evolution semiflows, as particular cases of skew-evolution cocycles over semiflows associated with a generalized dynamical system, are given in [18].

\section{On Classes of Dichotomies and Splitting Concepts}

In this section, we define several concepts of exponential dichotomy for skew-evolution semiflows. Also, we will establish some connections between these concepts and we will emphasize that they are not equivalent. In what follows, we will consider a skew-evolution semiflow $C=(\varphi, \Phi)$, where $\varphi$ is a semiflow and $\Phi$ is a skew-evolution cocycle.

Let $P: \mathbb{R}_{+} \rightarrow \mathscr{B}(V)$ be a family of projections on $V$. Then the mapping $Q: \mathbb{R}_{+} \rightarrow \mathscr{B}(V)$, given by $Q(t)=I-P(t)$, is also a family of projections on $V$, called the complementary family of projections relative to $P$. In what follows, we will denote

$$
\begin{aligned}
\operatorname{Ker} P(t) & =\{v \in V: P(t) v=0\}, \\
\text { Range } P(t) & =\{P(t) v: v \in V\} .
\end{aligned}
$$

Definition 9. A family of projections $P: \mathbb{R}_{+} \rightarrow \mathscr{B}(V)$ is said to be bounded if there exists a constant $M>0$ such that

$$
\|P(t)\| \leq M, \quad \forall t \geq 0
$$

Definition 10. We say that the family of projections $P: \mathbb{R}_{+} \rightarrow$ $\mathscr{B}(V)$ is compatible with the skew-evolution semiflow $\stackrel{+}{C}=$ $(\varphi, \Phi)$, if

$$
\begin{aligned}
& P(t) \Phi(t, s, \varphi(t, s, x))=\Phi(t, s, x) P(s), \\
& \forall(t, s, x) \in \Delta \times X .
\end{aligned}
$$

Remark 11. (i) If the family of projections $P: \mathbb{R}_{+} \rightarrow$ $\mathscr{B}(V)$ is compatible with the skew-evolution semiflow $C=$ $(\varphi, \Phi)$, then the complementary family of projections $Q$ is also compatible with $C$.

(ii) If the evolution cocycle $\Phi$ is reversible (i.e., $\Phi(t, s, \cdot)$ is bijective for all $(t, s) \in \Delta)$, then

$$
\begin{aligned}
& Q(s) \Phi(t, s, \varphi(t, s, x))^{-1}=\Phi(t, s, x)^{-1} Q(t), \\
& \forall(t, s, x) \in \Delta \times X .
\end{aligned}
$$

Definition 12. We say that the skew-evolution semiflow $C=$ $(\varphi, \Phi)$ generates a uniform exponential splitting (u.e.s.) if there exist a bounded family of projections $P: \Delta \rightarrow \mathscr{B}(V)$ compatible with $C$ and some constants $N \geq 1$ and $\alpha, \beta \in \mathbb{R}$ with $\alpha<\beta$, such that

$$
\begin{aligned}
& \left(s_{1}\right)\|P(t) \Phi(t, s, \varphi(t, s, x)) v\| \leq N e^{\alpha(t-s)}\|P(s) v\|, \\
& \left(s_{2}\right) N\|Q(t) \Phi(t, s, \varphi(t, s, x)) v\| \geq e^{\beta(t-s)}\|Q(s) v\|,
\end{aligned}
$$

for all $(t, s, x, v) \in \Delta \times Y$. The constants $\alpha$ and $\beta$ are called the growth rates of the skew-evolution semiflow $C$.

Remark 13. In the particular case when $\alpha<0<\beta$, the skew-evolution semiflow $C=(\varphi, \Phi)$ is called uniformly exponentially dichotomic (u.e.d.). Hence, we say that $C$ is (u.e.d.) if there exist a bounded family of projections $P: \Delta \rightarrow$ $\mathscr{B}(V)$ compatible with $C$ and some constants $N \geq 1$ and $\nu>0$ such that

$$
\begin{aligned}
& \left(d_{1}\right)\|P(t) \Phi(t, s, \varphi(t, s, x)) v\| \leq N e^{-v(t-s)}\|P(s) v\|, \\
& \left(d_{2}\right) N\|Q(t) \Phi(t, s, \varphi(t, s, x)) v\| \geq e^{v(t-s)}\|Q(s) v\|,
\end{aligned}
$$

for all $(t, s, x, v) \in \Delta \times Y$.

Indeed, for the necessity, it is convenient to take $v=$ $\min \{-\alpha, \beta\}$. The converse is obvious. 
The relation between the property of uniform exponential splitting and uniform exponential dichotomy is given by Proposition 14.

Proposition 14. The skew-evolution semiflow $C=(\varphi, \Phi)$ generates a uniform exponential splitting, with growth rates $\alpha$ and $\beta$, if and only if the $(\alpha+\beta) / 2$-shifted skew-evolution semiflow is uniformly exponentially dichotomic.

Proof. It is sufficient to observe that, according to Remark 4, conditions $\left(s_{1}\right)$ and $\left(s_{2}\right)$ are equivalent with

$$
\begin{gathered}
\left\|P(t) \Phi_{(\alpha+\beta) / 2}(t, s, \varphi(t, s, x)) v\right\| \\
\leq N e^{-((\beta-\alpha) / 2)(t-s)}\|P(s) v\|,
\end{gathered}
$$

respectively;

$$
\begin{aligned}
& N\left\|Q(t) \Phi_{(\alpha+\beta) / 2}(t, s, \varphi(t, s, x)) v\right\| \\
& \leq e^{((\beta-\alpha) / 2)(t-s)}\|Q(s) v\|,
\end{aligned}
$$

for all $(t, s, x, v) \in \Delta \times Y$. From Remark 13, the conclusion is obtained.

In the particular case of reversible evolution cocycles, we prove Proposition 15.

Proposition 15. Let $C=(\varphi, \Phi)$ be a skew-evolution semiflow with reversible evolution cocycle. Then $C$ generates a uniform exponential splitting, if and only if there exist a family of projections $P: \Delta \rightarrow \mathscr{B}(V)$ compatible with $C$ and some constants $\widetilde{N} \geq 1$ and $\alpha, \beta \in \mathbb{R}$ with $\alpha<\beta$, such that

$$
\begin{aligned}
& \left(s_{1}^{\prime}\right)\|P(t) \Phi(t, s, \varphi(t, s, x))\| \leq \widetilde{N} e^{\alpha(t-s)}, \\
& \left(s_{2}^{\prime}\right)\left\|\Phi(t, s, x)^{-1} Q(t)\right\| \leq \widetilde{N} e^{-\beta(t-s)},
\end{aligned}
$$

for all $(t, s, x) \in \Delta \times X$.

Proof.

Necessity. Suppose that $C$ has the property of uniform exponential splitting. Then, by Definition 12, we have that

$$
\begin{aligned}
& \|P(t) \Phi(t, s, \varphi(t, s, x))\|=\sup _{\|v\| \leq 1}\|\Phi(t, s, x) P(s) v\| \\
& \leq N e^{\alpha(t-s)} \sup _{\|v\| \leq 1}\|P(s) v\| \leq N e^{\alpha(t-s)}\|P(s)\| \\
& \leq N(M+1) e^{\alpha(t-s)},
\end{aligned}
$$

for all $(t, s, x) \in \Delta \times X$, as $P$ is a bounded family of projections. Hence, condition $\left(s_{1}^{\prime}\right)$ is satisfied for $\widetilde{N}=N(M+1)$.

Similarly,

$$
\begin{gathered}
\left\|\Phi(t, s, x)^{-1} Q(t)\right\|=\sup _{\|v\| \leq 1}\left\|\Phi(t, s, x)^{-1} Q(t) v\right\| \\
=\sup _{\|v\| \leq 1}\left\|Q(s) \Phi(t, s, \varphi(t, s, x))^{-1} v\right\| \leq N e^{-\beta(t-s)} \\
\quad \cdot \sup _{\|v\| \leq 1}\left\|\Phi(t, s, x) Q(s) \Phi(t, s, \varphi(t, s, x))^{-1} v\right\|
\end{gathered}
$$

$$
\begin{aligned}
& =N e^{-\beta(t-s)} \sup _{\|v\| \leq 1} \| Q(t) \Phi(t, s, \varphi(t, s, x)) \\
& \cdot \Phi(t, s, \varphi(t, s, x))^{-1} v\left\|=N e^{-\beta(t-s)} \sup _{\|v\| \leq 1}\right\| Q(t) v \| \\
& \leq N(M+1) e^{-\beta(t-s)}=\widetilde{N} e^{-\beta(t-s)},
\end{aligned}
$$

for all $(t, s, x) \in \Delta \times X$, and, hence, $\left(s_{2}^{\prime}\right)$ is obtained.

Sufficiency. If inequalities $\left(s_{1}^{\prime}\right)$ and $\left(s_{2}^{\prime}\right)$ are satisfied, then

$$
\begin{aligned}
& \|P(t) \Phi(t, s, \varphi(t, s, x)) v\| \\
& \quad \leq\|\Phi(t, s, x) P(s)\|\|P(s) v\| \leq \widetilde{N} e^{\alpha(t-s)}\|P(s) v\|, \\
& \|Q(s) v\|=\left\|\Phi(t, s, x)^{-1} \Phi(t, s, x) Q(s) v\right\| \\
& \quad=\left\|\Phi(t, s, x)^{-1} Q(t) \Phi(t, s, \varphi(t, s, x)) v\right\| \\
& \leq \widetilde{N} e^{-\beta(t-s)}\|Q(t) \Phi(t, s, \varphi(t, s, x)) v\|,
\end{aligned}
$$

for all $(t, s, x, v) \in \Delta \times Y$.

Finally, we obtain that conditions $\left(s_{1}\right)$ and $\left(s_{2}\right)$ are satisfied for the constants $\widetilde{N}, \alpha$, and $\beta$. Moreover,

$$
\|P(t)\|=\|\Phi(t, t, x) P(t)\| \leq \widetilde{N}, \quad \forall t \geq 0,
$$

which shows that the family of projections $P$ is bounded. Thus, $C$ generates a uniform exponential splitting.

It is a natural question if the family of projections $P$ from Definition 12 is unique. In this purpose we prove Lemma 16.

Lemma 16. Let $P, R: \Delta \rightarrow \mathscr{B}(V)$ be two family of projections with the property RangeP $(t)=$ RangeR $(t), \forall t \geq 0$. If $Q(t)=$ $I-P(t)$ and $S(t)=I-R(t)$ are the complementary families of $P$ and $R$, respectively, then, for every $t \geq 0$, the following equalities are satisfied:

(i) $P(t)=R(t) P(t)$.

(ii) $R(t)=P(t) R(t)$.

(iii) $Q(t)=Q(t) S(t)=[I+R(t)-P(t)] S(t)$.

(iv) $S(t)=S(t) Q(t)=[I+P(t)-R(t)] Q(t)$.

Proof. (i) For $v \in V$, there exists $u \in V$ such that $P(t) v=$ $R(t) u$ and, hence,

$$
R(t) P(t) v=R^{2}(t) u=R(t) u=P(t) v, \quad \forall t \geq 0 .
$$

(ii) Similarly, for every $v \in V$, there exists $u \in V$ with $R(t) v=P(t) u$, which implies that

$$
P(t) R(t) v=P^{2}(t) u=P(t) u=R(t) v, \quad \forall t \geq 0 .
$$

(iii) We have, for all $t \geq 0$,

$$
\begin{aligned}
{[I} & +R(t)-P(t)] S(t)=[Q(t)+R(t)] S(t) \\
& =Q(t) S(t)=[I-P(t)][I-R(t)] \\
& =I-R(t)-P(t)+R(t)=I-P(t)=Q(t) .
\end{aligned}
$$


(iv) We obtain, for all $t \geq 0$,

$$
\begin{aligned}
S(t) & =I-R(t)=[I-R(t)][I-P(t)]=S(t) Q(t) \\
& =[S(t)+P(t)] Q(t)=[I+P(t)-R(t)] Q(t) .
\end{aligned}
$$

Now, we prove Proposition 17.

Proposition 17. Let $P, R: \Delta \rightarrow \mathscr{B}(V)$ be two bounded families of projections compatible with the skew-evolution semiflow $C$ with the property Range $P(t)=\operatorname{Range} R(t), \forall t \geq 0$. If $C$ has uniform exponential splitting relative to the family of projections $P$, then it also generates a uniform exponential splitting relative to $R$.

Proof. Suppose that $C$ has uniform exponential splitting with respect to the bounded family of projections $P$ and let $R$ be a bounded family of projections compatible with $C$ such that Range $R(t)=$ Range $P(t), \forall t \geq 0$. Let $M>0$ be a constant such that $\|P(t)\|+\|R(t)\| \leq M, \forall t \geq 0$.

Then, according to Definition 12 and Lemma 16, we obtain

$$
\begin{gathered}
\|R(t) \Phi(t, s, \varphi(t, s, x)) v\|=\|\Phi(t, s, x) P(s) R(s) v\| \\
\leq N e^{\alpha(t-s)}\|P(s) R(s) v\| \leq M N e^{\alpha(t-s)}\|R(s) v\|,
\end{gathered}
$$

for all $(t, s, x, v) \in \Delta \times Y$.

Similarly,

$$
\begin{gathered}
\|S(s) v\| \leq\|I+P(s)-R(s)\|\|Q(s) v\| \leq(1+2 M) \\
\cdot\|Q(s) v\| \leq N(1+2 M) e^{-\beta(t-s)} \| Q(t) \\
\cdot \Phi(t, s, \varphi(t, s, x)) v \| \leq N(1+2 M) \\
\cdot e^{-\beta(t-s)}\|\Phi(t, s, x)[I+R(s)-P(s)] S(s) v\| \\
=N(1+2 M) e^{-\beta(t-s)} \|[I+R(t)-P(t)] \\
\cdot \Phi(t, s, \varphi(t, s, x)) S(s) v \| \leq N(1+2 M)^{2} \\
\cdot e^{-\beta(t-s)}\|\Phi(t, s, x) S(s) v\|,
\end{gathered}
$$

for all $(t, s, x, v) \in \Delta \times Y$.

Finally, we obtain that $C$ generates a uniform exponential splitting with respect to the family of projections $R$.

Proposition 18. If the skew-evolution semiflow $C=(\varphi, \Phi)$ generates a uniform exponential splitting, then there exists a bounded family of projections $P: \Delta \rightarrow \mathscr{B}(V)$ compatible with $C$ such that for every $(t, s, x) \in \Delta \times X$ the restriction of $\Phi(t, s, x)$ to $\operatorname{Ker} P(s)$ is injective.

Proof. Indeed, if $C$ generates uniform exponential splitting with respect to the family of projections $P$ and $v \in \operatorname{Ker} P(t)$ with $\Phi(t, s, x) v=0$, then, according to Definition 12, we have

$$
\begin{aligned}
e^{\beta(t-s)}\|v\| & =e^{\beta(t-s)}\|Q(s) v\| \\
& \leq N\|Q(t) \Phi(t, s, \varphi(t, s, x)) v\|
\end{aligned}
$$

$$
\begin{aligned}
& =N\|\Phi(t, s, x) Q(s) v\|=N\|\Phi(t, s, x) v\| \\
& =0
\end{aligned}
$$

and, hence, $v=0$.

\section{Main Results}

We intend to prove in this section, according to Proposition 18, that it comes naturally to give a sufficient condition; in order to obtain that, for every $(t, s, x) \in \Delta \times X$, the evolution cocycle $\Phi$ is an isomorphism from $\operatorname{Ker} P(s)$ to $\operatorname{Ker} P(t)$. To this aim, we give Definition 19.

Definition 19. A family of projections $P: \Delta \rightarrow \mathscr{B}(V)$ is called strongly compatible with a skew-evolution semiflow $C=(\varphi, \Phi)$ if it is compatible with $C$, and, for all $(t, s, x, y) \in$ $\Delta \times X \times \operatorname{Ker} P(t)$, there exists $v \in V$ such that $y=\Phi(t, s, x) v$.

Remark 20. If the family of projections $P: \Delta \rightarrow \mathscr{B}(V)$ is compatible with the skew-evolution semiflow $C=(\varphi, \Phi)$, then it is strongly compatible with $C$ if and only if

$$
\operatorname{Ker} P(t) \subset \text { Range } \Phi(t, s, x), \quad \forall(t, s, x) \in \Delta \times X
$$

Remark 21. If the family of projections $P: \Delta \rightarrow \mathscr{B}(V)$ is compatible with the skew-evolution semiflow $C=(\varphi, \Phi)$, with the property that the evolution cocycle $\Phi$ is reversible, then it is also strongly compatible with $C$.

Indeed, for $y \in \operatorname{Ker} P(t), y=\Phi(t, s, x) \Phi(t, s, x)^{-1} y \epsilon$ Range $\Phi(t, s, x), \forall(t, s, x) \in \Delta \times X$.

Definition 22. We say that a skew-evolution semiflow $C=$ $(\varphi, \Phi)$ induces a strong uniform exponential splitting (s.u.e.s.) if there exists a family of projections $P: \Delta \rightarrow \mathscr{B}(V)$ strongly compatible with $C$ such that $C$ generates a uniform exponential splitting with respect to $P$.

In particular, if $C$ is uniformly exponentially dichotomic with respect to a family of projections $P: \Delta \rightarrow \mathscr{B}(V)$ which is strongly compatible with $C$, then $C$ is called strongly uniformly exponentially dichotomic (s.u.e.d.).

It is obvious that

$$
\begin{aligned}
& (\text { s.u.e.s. }) \Longrightarrow(\text { u.e.s. }) \\
& \text { (s.u.e.d. }) \Longrightarrow(\text { u.e.d. })
\end{aligned}
$$

The converse implications are not true, fact illustrated by Example 23.

Example 23. Let us consider the differential equation on a Banach space:

$$
\dot{v}(t)=f(t) v(t), \quad t \geq 0,
$$

where $f: \mathbb{R}_{+} \rightarrow \mathbb{R}_{+}$is a decreasing function with $\lim _{t \rightarrow \infty} f(t)=l>0$ and let $\lambda>f(0)$. We consider the metric space $(X, d)$ and the evolution semiflow $\varphi$ on $X$ defined as in Example 7. 
Let $V=\mathbb{R}^{3}$ with the norm $\left\|\left(v_{1}, v_{2}, v_{3}\right)\right\|=\left\|v_{1}\right\|+\left\|v_{2}\right\|+$ $\left\|v_{3}\right\|$. We define the evolution cocycle $\Phi: \Delta \times X \rightarrow \mathscr{B}(V)$ by

$$
\begin{gathered}
\Phi(t, s, x) v=\left(v_{1} e^{-\lambda(t-s)+\int_{s}^{t} x(\tau-s) d \tau},\right. \\
\left.v_{2}[\psi(s)]^{t-s+\int_{s}^{t} x(\tau-s) d \tau}, v_{3} e^{(t-s) x(0)+\int_{s}^{t} x(\tau-s) d \tau}\right),
\end{gathered}
$$

where

$$
\psi(s)= \begin{cases}0, & s=0 \\ e, & s>0\end{cases}
$$

It is a simple verification that

$$
\begin{gathered}
P: \Delta \longrightarrow \mathscr{B}(V), \\
P(t)\left(v_{1}, v_{2}, v_{3}\right)= \begin{cases}\left(v_{1}, v_{2}, 0\right), & t=0 \\
\left(v_{1}+v_{2} e^{-2 \lambda t}, 0,0\right), & t>0\end{cases}
\end{gathered}
$$

is a bounded family of projections compatible with $C$. The complementary family of projections of $P$ is

$$
\begin{gathered}
Q: \Delta \longrightarrow \mathscr{B}(V), \\
Q(t)\left(v_{1}, v_{2}, v_{3}\right)= \begin{cases}\left(0,0, v_{3}\right), & t=0 \\
\left(-v_{2} e^{-2 \lambda t}, v_{2}, v_{3}\right), & t>0 .\end{cases}
\end{gathered}
$$

We observe that, for all $(t, s, x) \in \Delta \times X$ and $v \in V$, we have

$$
\begin{aligned}
& \Phi(t, s, x) P(s) v \\
& = \begin{cases}\left(v_{1} e^{-\lambda t+\int_{0}^{t} x(\tau) d \tau}, 0,0\right), & t \geq s=0 \\
\left(\left(v_{1}+v_{2} e^{-2 \lambda s}\right) e^{-\lambda(t-s)+\int_{s}^{t} x(\tau-s) d \tau}, 0,0\right), & t \geq s>0 \\
\left(v_{1}, v_{2}, 0\right), & t=s=0,\end{cases} \\
& \Phi(t, s, x) Q(s) v \\
& = \begin{cases}\left(0,0, v_{3} e^{x(0) t+\int_{0}^{t} x(\tau) d \tau}\right), & t \geq s=0 \\
\left(-v_{2} e^{-\lambda t-\lambda s}, v_{2} e^{t-s}, v_{3} e^{(t-s) x(0)}\right) e^{\int_{s}^{t} x(\tau-s) d \tau}, & t \geq s>0 \\
\left(0,0, v_{3}\right), & t=s=0 .\end{cases}
\end{aligned}
$$

The following relations hold:

$$
\begin{aligned}
& \|P(t) \Phi(t, s, \varphi(t, s, x)) v\|=\|\Phi(t, s, x) P(s) v\| \\
& \quad \leq e^{-[\lambda-x(0)](t-s)}\|P(s) v\|, \\
& \|Q(t) \Phi(t, s, \varphi(t, s, x)) v\|=\|\Phi(t, s, x) Q(s) v\| \\
& \quad \geq e^{l(t-s)}\|Q(s) v\|,
\end{aligned}
$$

for all $(t, s, x, v) \in \Delta \times Y$.

Thus, we obtain that $C$ has uniform exponential dichotomy and, further, generates uniform exponential splitting with respect to the family of projections $P$.
On the other hand, $C$ is not strongly uniformly exponentially dichotomic with respect to $P$ because if, for example,

$$
y=\left(-\frac{1}{e^{2}}, 1,0\right) \in \operatorname{Ker} P(s)
$$

we have that $y \notin$ Range $\Phi(1,0, x)$, because if we suppose that there exists $v=\left(v_{1}, v_{2}, v_{3}\right) \in \mathbb{R}^{3}$ with $\Phi(1,0, x) v=y$, then

$$
\left(-\frac{1}{e^{2}}, 1,0\right)=\left(\frac{v_{1}}{e}, 0, v_{3} e\right) \text {, }
$$

which is a contradiction.

Theorem 24. If a skew-evolution semiflow $C=(\varphi, \Phi)$ induces a strong uniform exponential splitting with respect to a family of projections $P: \Delta \rightarrow \mathscr{B}(V)$, then, for all $(t, s, x) \in \Delta \times X$, the bounded evolution cocycle $\Phi$ (i.e., there exists $M>0$ such that $\|\Phi(t, s, x)\| \leq M, \forall(t, s, x) \in \Delta \times X)$ is an isomorphism from $\operatorname{Ker} P(s)$ to $\operatorname{Ker} P(t)$.

Proof. According to Proposition 18, it is sufficient to prove that, for all $(t, s, x) \in \Delta \times X$ and $y \in \operatorname{Ker} P(t)$, there exists $v \in \operatorname{Ker} P(s)$ such that $\Phi(t, s, x) v=y$. Indeed, if $y \in \operatorname{Ker} P(t)$, then $y=Q(t) y$ and there exists $v_{0} \in V$ such that $y=$ $\Phi(t, s, x) v_{0}$, which implies that

$$
\begin{aligned}
y & =Q(t) y=Q(t) \Phi(t, s, \varphi(t, s, x)) v_{0} \\
& =\Phi(t, s, x) Q(s) v_{0}=\Phi(t, s, x) v,
\end{aligned}
$$

for all $(t, s, x) \in \Delta \times X$, where $v=Q(s) v_{0} \in \operatorname{Ker} P(s)$.

As a particular case, we obtain Corollary 25.

Corollary 25. If a skew-evolution semiflow $C=(\varphi, \Phi)$ is strongly uniformly exponentially dichotomic with respect to a family of projections $P: \Delta \rightarrow \mathscr{B}(V)$, then, for all $(t, s, x) \in$ $\Delta \times X$, the bounded evolution cocycle $\Phi$ is an isomorphism from $\operatorname{Ker} P(s)$ to $\operatorname{Ker} P(t)$.

\section{Conclusions}

Dynamical systems theory deals with the analysis of the long-term qualitative behavior. One of the central interests in the asymptotic behavior of dynamical systems is to find conditions for their solutions to be stable, unstable, or exponentially dichotomic.

The concept of exponential splitting is stronger than the exponential dichotomy, but they are equivalent under suitable conditions, for example,

(i) $P$ bounded,

(ii) the skew-evolution cocycle with uniform exponential growth and $\beta>0$.

We prefer to deal rather with the notion of exponential splitting than exponential dichotomy in view of future applications to nonlinear equations, as we intend to obtain formulae for various growth rates and bounds in the more general case of exponential splitting. 
As applications of the splitting theorems, we intend to propose necessary and sufficient conditions of input-output type for the exponential stability of autonomous systems as well as consequences which allow us to establish connections between stability and stabilizability and detectability, respectively. They are also useful in the study of exponential expansivity of control systems, with applications in engineering.

\section{Competing Interests}

The author declares that there are no competing interests regarding the publication of this article.

\section{Acknowledgments}

The author gratefully acknowledges helpful support and comments from Professor Emeritus Mihail Megan, the Head of the Research Seminar on Mathematical Analysis and Applications in Control Theory at the West University of Timişoara, Romania.

\section{References}

[1] C. Chicone and Y. Latushkin, Evolution Semigroups in Dynamical Systems and Differential Equations, vol. 70 of Mathematical Surveys and Monographs, American Mathematical Society, Providence, RI, USA, 1999.

[2] G. R. Sell and Y. You, Dynamics of evolutionary equations, vol. 143 of Applied Mathematical Sciences, Springer-Verlag, 2002.

[3] O. Perron, "Die Stabilitätsfrage bei Differentialgleichungen," Mathematische Zeitschrift, vol. 32, no. 1, pp. 703-728, 1930.

[4] J. L. Daleckii and M. G. Krein, Stability of Solutions of Differential Equations in Banach Spaces, vol. 43 of Translations of Mathematical Monographs, American Mathematical Society, Providence, RI, USA, 5th edition, 1974.

[5] J. L. Massera and J. J. Schäffer, Linear differential equations and function spaces, vol. 21 of Pure and Applied Mathematics, Academic Press, London, UK, 1966.

[6] Y. Latushkin, S. Montgomery-Smith, and T. Randolph, "Evolutionary semigroups and dichotomy of linear skew-product flows on locally compact spaces with Banach fibers," Journal of Differential Equations, vol. 125, no. 1, pp. 73-116, 1996.

[7] R. J. Sacker and G. R. Sell, "Existence of dichotomies and invariant splittings for linear differential systems. I," Journal of Differential Equations, vol. 15, pp. 429-458, 1974.

[8] B. Aulbach and J. Kalkbrenner, "Exponential forward splitting for noninvertible difference equations," Computers \& Mathematics with Applications, vol. 42, no. 3-5, pp. 743-754, 2001.

[9] B. Aulbach and S. Siegmund, "The dichotomy spectrum for noninvertible systems of linear difference equations," Journal of Difference Equations and Applications, vol. 7, no. 6, pp. 895-913, 2001.

[10] J. Kalkbrenner, Exponentielle Dichotomie und Chaotische Dynamik Nichtinvertierbarer Differenzengleichungen, Verlegt bei Dr. Bernd Wissner, Augsburg, Germany, 1994.

[11] H. Leiva, E. Medina, and N. Merentes, "Relative asymptotic equivalence between difference equations," Journal of Difference Equations and Applications, vol. 21, no. 5, pp. 418-436, 2015.
[12] S.-N. Chow and H. Leiva, "Existence and roughness of the exponential dichotomy for skew-product semiflow in Banach spaces," Journal of Differential Equations, vol. 120, no. 2, pp. 429$477,1995$.

[13] M. Megan and C. Stoica, "Exponential instability of skewevolution semiflows in Banach spaces," Studia Universitatis Babes-Bolyai. Mathematica, vol. 53, no. 1, pp. 17-24, 2008.

[14] M. Megan and C. Stoica, "On uniform exponential trichotomy of evolution operators in Banach spaces," Integral Equations and Operator Theory, vol. 60, no. 4, pp. 499-506, 2008.

[15] C. Stoica, Uniform Asymptotic Behaviors for Skew-Evolution Semiflows on Banach Spaces, Mirton Publishing House, Timişoara, Romania, 2010.

[16] C. Stoica, "Trichotomy for dynamical systems in banach spaces," The Scientific World Journal, vol. 2013, Article ID 793813, 8 pages, 2013.

[17] C. Stoica, "Approaching the discrete dynamical systems by means of skew-evolution semiflows," Discrete Dynamics in Nature and Society, vol. 2016, Article ID 4375069, 10 pages, 2016.

[18] C. Stoica and M. Megan, "On uniform exponential stability for skew-evolution semiflows on Banach spaces," Nonlinear Analysis: Theory, Methods \& Applications, vol. 72, no. 3-4, pp. 1305-1313, 2010.

[19] C. Stoica and M. Megan, "On (h,k)-trichotomy for skewevolution semiflows in Banach spaces," Studia Universitatis Babes-Bolyai. Mathematica, vol. 56, no. 4, pp. 147-156, 2002.

[20] C. Stoica, "Exponential dichotomy versus exponential splitting for skew-evolution semiflows in infinite dimensional spaces," ATINER's Conference Paper Series FOR2015-1744, 2015.

[21] A. J. Bento and C. M. Silva, "Nonuniform dichotomic behavior: Lipschitz invariant manifolds for ODEs," Bulletin des Sciences Mathématiques, vol. 138, no. 1, pp. 89-109, 2014.

[22] P. V. Hai, "Continuous and discrete characterizations for the uniform exponential stability of linear skew-evolution semiflows," Nonlinear Analysis: Theory, Methods \& Applications, vol. 72, no. 12, pp. 4390-4396, 2010.

[23] P. V. Hai, "Discrete and continuous versions of Barbashintype theorem of linear skew-evolution semiflows," Applicable Analysis, vol. 90, no. 12, pp. 1897-1907, 2011.

[24] T. Yue, X. Q. Song, and D. Q. Li, "On weak exponential expansiveness of skew-evolution semiflows in Banach spaces," Journal of Inequalities and Applications, vol. 2014, article 165, 2014.

[25] S.-N. Chow and H. Leiva, "Two definitions of exponential dichotomy for skew-product semiflow in Banach spaces," Proceedings of the American Mathematical Society, vol. 124, no. 4, pp. 1071-1081, 1996.

[26] H. Leiva and H. Rodrigues, "Relative asymptotic equivalence of evolution equations," Nonlinear Analysis: Theory, Methods \& Applications, vol. 47, no. 7, pp. 4579-4590, 2001.

[27] T. Caraballo, J. A. Langa, and J. Valero, "Global attractors for multivalued random dynamical systems," Nonlinear Analysis: Theory, Methods \& Applications, vol. 48, no. 6, pp. 805-829, 2002.

[28] J. Valero, "About non-autonomous multivalued dynamical systems and their applications to reaction-diffusion equations," in Proceedings of the Joint SIAM/RSME-SCM Meeting on Emerging Topics in Dynamical Systems and Partial Differential Equations (DSPDEs '10), Barcelona, Spain, 2010. 
[29] C. Stoica, "Cocycles over non-autonomous dynamical systems in Banach spaces," in Essays on Mathematics and Statistics: Volume 5, pp. 65-72, Athens Institute for Education and Research, 2015.

[30] C. Stoica and L. Biriş, "Nonuniform behaviors for cocycles over multivalued non-autonomous dynamical systems in Banach spaces," in Proceedings of the 13th International Conference on Mathematics and its Applications (ICMA '12), pp. 131-136, 2013. 


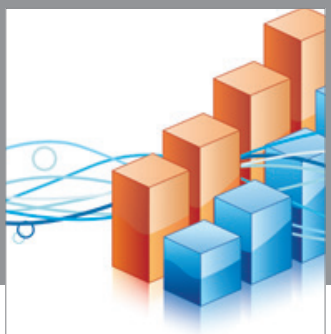

Advances in

Operations Research

vatem alat4

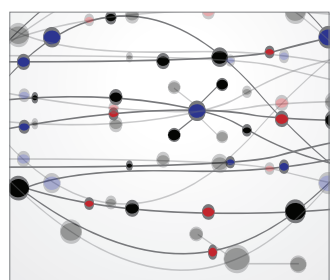

\section{The Scientific} World Journal
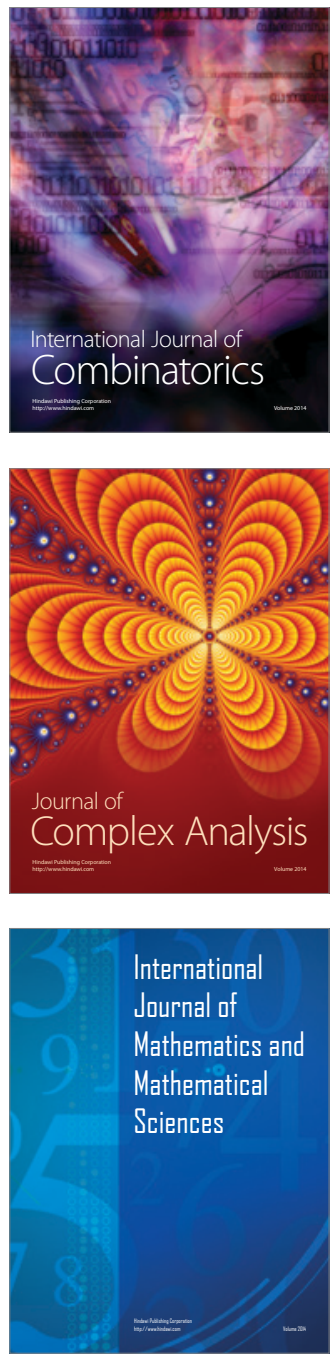
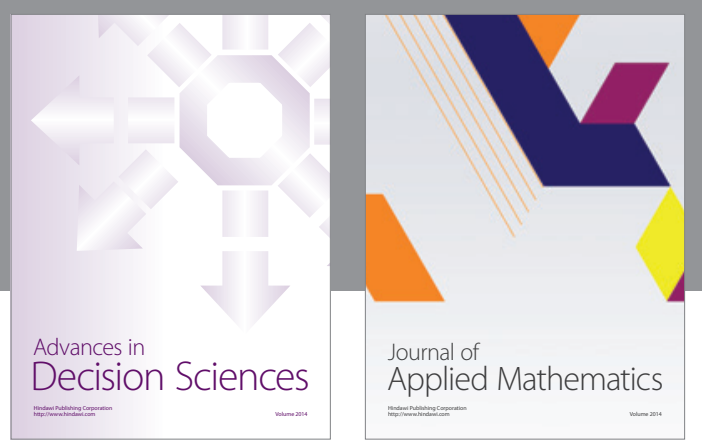

Algebra

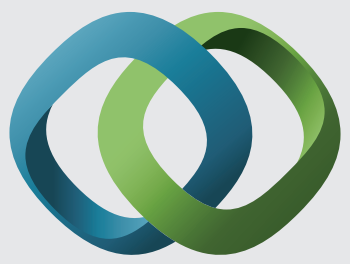

\section{Hindawi}

Submit your manuscripts at

https://www.hindawi.com
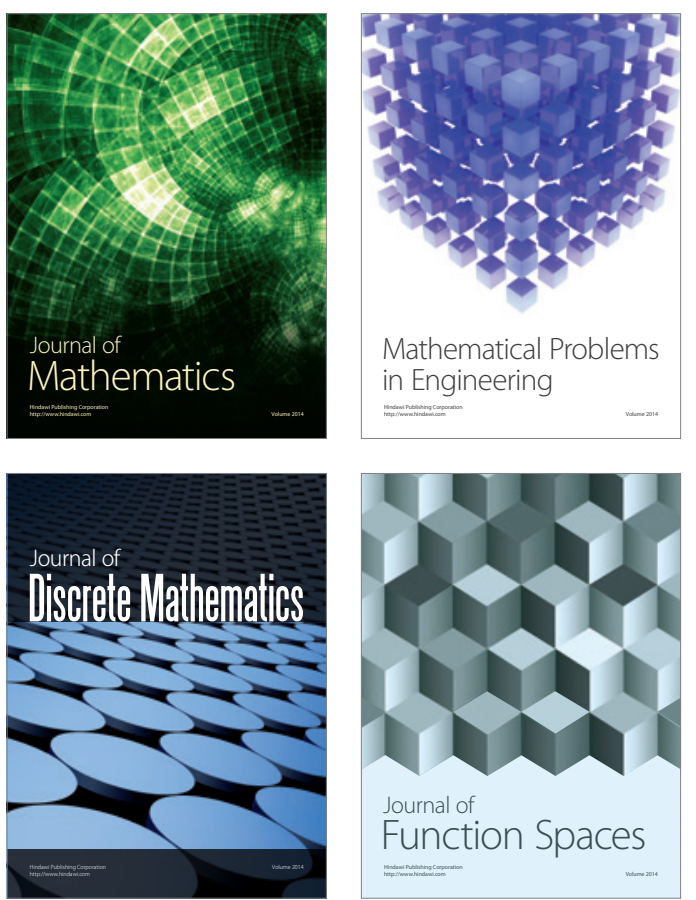

Mathematical Problems in Engineering
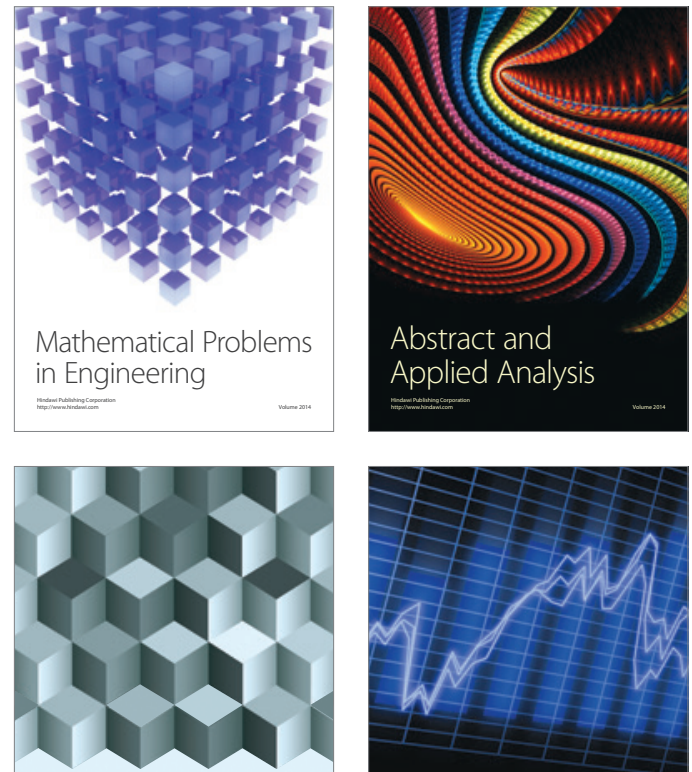

Journal of

Function Spaces

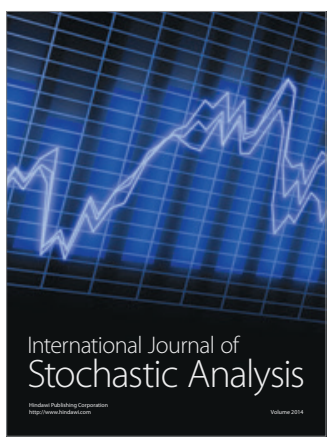

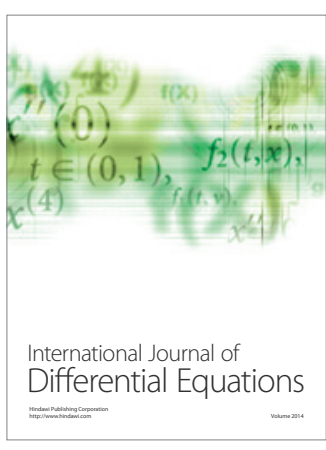
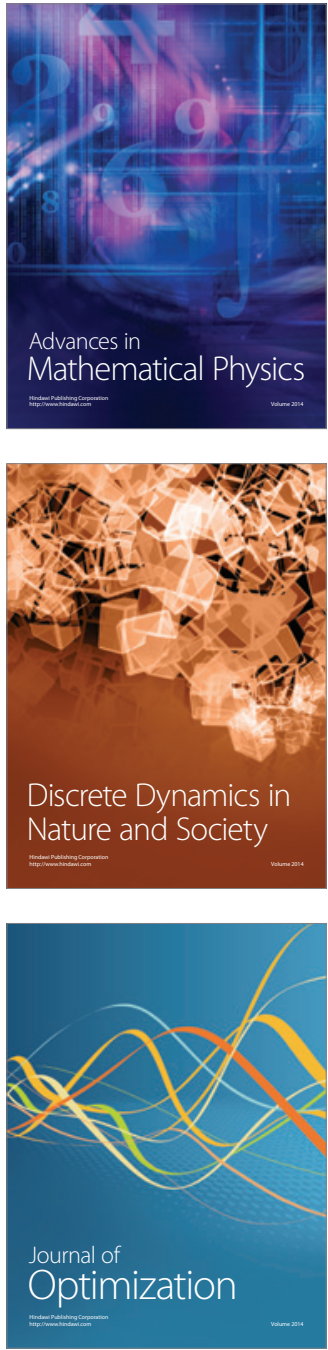